\title{
Electromagnetic design of superconducting dipoles based on sector coils
}

\author{
L. Rossi and Ezio Todesco \\ Accelerator Technology Department, CERN, CH-1211 Geneva, Switzerland
}

(Received 2 August 2007; published 30 November 2007)

\begin{abstract}
We study the coil layouts of superconducting dipoles for particle accelerators based on the sector geometry. We show that a simple model based on a sector coil with a wedge allows us to derive an equation giving the short sample field as a function of the aperture, coil width, cable properties, and superconducting material. The equation agrees well with the actual results of several dipole coils that have been built in the past 30 years. The improvements due to the grading technique and the iron yoke are also studied. The proposed equation can be used as a benchmark to judge the efficiency of the coil design, and to carry out a global optimization of an accelerator layout.
\end{abstract}

DOI: 10.1103/PhysRevSTAB.10.112401

\section{INTRODUCTION}

Superconducting dipoles have been used for 30 years to bend particle beams in accelerator machines. Fields of 4 to $10 \mathrm{~T}$ have been reached with electromagnets based on $\mathrm{Nb}-$ Ti cables [1-7], whose critical field at $1.9 \mathrm{~K}$ is around $13 \mathrm{~T}$. A new generation based on the $\mathrm{Nb}_{3} \mathrm{Sn}$ has allowed to break the $10 \mathrm{~T}$ barrier, such as the CERN-Elin [8], the MSUT [9] of the Twente University-CERN (11 T), and the D20 [10] made in Lawrence Berkeley Laboratory (13 T). Tests of a more recent $\mathrm{Nb}_{3} \mathrm{Sn}$ conductor in a block configuration magnet $[11,12]$ showed that the material can reach $16 \mathrm{~T}$ (LBNL HDI), and programs to obtain large fields in acceleratorlike magnets are ongoing [12-14]. This technology would have the potential to double the energy of the Large Hadron Collider (LHC).

The simplest way to create a pure dipolar field is to have an annulus where the current density is proportional to the cosine of the azimuth ( $\cos \theta$ coil). In practical layouts, the current density has to be constant and conductors are piled up in blocks separated by spacers. This arrangement aims at approximating a $\cos \theta$ coil with a finite number of blocks carrying the same constant current density. One defines a $\cos \theta$ layout when the shape of the coil is still an annulus, thus providing a self-supporting structure, and the blocks are shaped as sectors. Most of the dipole coils have been based on this layout $[15,16]$, with different number of layers and of spacers. Alternative layouts are based on rectangular blocks [12].

The aim of this paper is to find approximate equations that provide the field reachable in a given aperture with a given quantity of conductor, and to estimate what are the most effective design options among the ones that have been chosen in the past. The approach is analogous to what has been done for the quadrupoles [17]: we carry out an exhaustive analysis of the sector coil models (i.e., $\cos \theta$ layouts), using semianalytical tools when the pure analytical approach is not viable.

The sector coil models are slightly more representative than the $\cos \theta$ coil (i.e. the ideal coil with current density
PACS numbers: 07.55.Db, 41.85.Lc, 84.71.Ba, 85.70.Ay

depending on the azimuth), which has been extensively studied in $[18,19]$, since they include the difference between central field and peak field in the coil. This difference is not negligible when the ratio between the coil width and the aperture radius is small, such as in the RHIC dipoles [4]. The extension of this analysis to alternative layouts such as the block coil or the common coil will be presented separately. The final aim of the work is to have handy formulas to be able to carry out a global optimization of the parameters of an accelerator or of a part of it, and a dimension/cost estimate.

In Sec. II we present the equations for the short sample field for the $\mathrm{Nb}-\mathrm{Ti}$ and $\mathrm{Nb}_{3} \mathrm{Sn}$. The analysis of the sector layouts is given in Sec. III, including a comparison between different designs, dependence on coil width and magnet apertures, and grading techniques. The analysis of the actual design of 11 dipoles is done in Sec. IV, and the impact of the iron is analyzed in Sec. V.

\section{EQUATIONS DEFINING THE SHORT SAMPLE FIELD}

\section{A. Critical current density}

$\mathrm{A} \mathrm{Nb}-\mathrm{Ti}$ or $\mathrm{Nb}_{3} \mathrm{Sn}$ filament carrying a current density $j_{\mathrm{sc}}$ in a magnetic field $B$ is superconducting as long as the current density is less than the critical current density $j_{\mathrm{sc}, c}$, which for the Nb-Ti can be fit using the expression [20]

$$
j_{\mathrm{sc}, c}=C(T) B^{\alpha-1}\left(1-\frac{B}{B_{c 2}^{*}(T)}\right)^{\beta},
$$

where the constant $C$ depends on the temperature, $\alpha \sim$ $0.5-1.0$ and $\beta \sim 1$, and $B^{*}{ }_{c 2}$ is the critical field at zero current density at the temperature $T$. This pretty complex parameter dependence can be very well approximated over a large domain by a simple linear function of the magnetic field (see Fig. 1):

$$
j_{\mathrm{sc}, c}=c(b-B), \quad B<b .
$$

The fit is good for values of the magnetic field larger than 


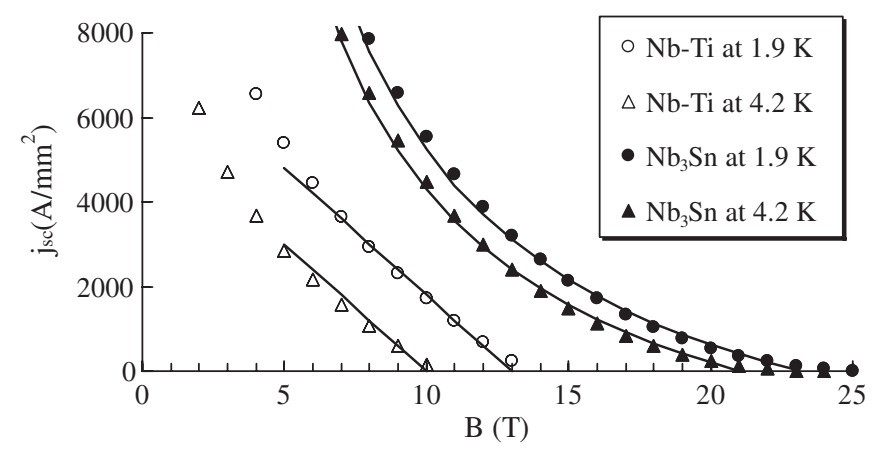

FIG. 1. Critical surface for Nb-Ti: fit of Eq. (1) (empty markers), and linear fit (solid line) of Eq. (2). Critical surface for $\mathrm{Nb}_{3} \mathrm{Sn}$ : fit of Eq. (3) (full markers), and hyperbolic fit (solid line) of Eq. (4).

$5 \mathrm{~T}$ at $1.9 \mathrm{~K}$, and $2 \mathrm{~T}$ at $4.2 \mathrm{~K}$, with $b \sim 10 \mathrm{~T}$ at $4.2 \mathrm{~K}$ and $13 \mathrm{~T}$ at $1.9 \mathrm{~K}$, and the slope $c \sim 6.00 \times 10^{8} \mathrm{~A} /\left(\mathrm{T} \mathrm{m}^{2}\right)$ is independent of the temperature. This corresponds to having $3000 \mathrm{~A} / \mathrm{mm}^{2}$ at $8 \mathrm{~T}$ and $1.9 \mathrm{~K}$, or at $5 \mathrm{~T}$ and $4.2 \mathrm{~K}$. We will show that this is the relevant domain for our analysis.

For the $\mathrm{Nb}_{3} \mathrm{Sn}$, the critical surface can be written according to Kramer [21]:

$$
j_{\mathrm{sc}, c}(B)=\frac{C(T, \varepsilon)}{\sqrt{B}}\left(1-\frac{B}{B_{c 2}^{*}(T, \varepsilon)}\right)^{2},
$$

where the two constants depend on temperature $T$ and strain $\varepsilon$.

In [17] we proposed an hyperbolic fit

$$
j_{\mathrm{sc}}=c\left(\frac{b}{B}-1\right) \quad B<b,
$$

where $b$ is the value of the critical field at zero current density according to the fit. The parametrization (4) agrees well with (3) for typical parameters on a very wide domain, see Fig. 1. For the case of a very high density current $\mathrm{Nb}_{3}$ Sn giving $3000 \mathrm{~A} / \mathrm{mm}^{2}$ at $12 \mathrm{~T}, 4.2 \mathrm{~K}$, with a deformation of $\varepsilon=0.003$, the agreement is within $5 \%$ from 5 to $17 \mathrm{~T}$ at $4.2 \mathrm{~K}$ (with $c=3.9 \times 10^{9}\left[\mathrm{~A} / \mathrm{m}^{2}\right]$ and $b=$ $21.0 \mathrm{~T}$ ). For the same case, at $1.9 \mathrm{~K}$ one has $c=4.0 \times$ $10^{9}\left[\mathrm{~A} / \mathrm{m}^{2}\right]$ and $b=23.1 \mathrm{~T}$. These cable performances correspond to the original aim of the LHC Accelerator Research Program [22] and of the Next European Dipole [14] conductor programs.

A practical superconductor wire is made of filaments in a copper matrix, and one defines $\nu_{\mathrm{Cu}-\mathrm{sc}}$ as the ratio between the quantity of copper (stabilizer) and the superconductor in the strand cross section. For Nb-Ti this is a straightforward formulation. On the other hand, $\mathrm{Nb}_{3} \mathrm{Sn}$ strands contain passive materials that are at the same time not superconductor and not used for stabilization: these elements are necessary to the formation of the superconductor itself. Since the critical current is referred to the surface of the nonstabilizer material (non- $\mathrm{Cu}$ ), a consistent definition in this case is $\nu_{\mathrm{Cu} \text {-sc }}$ as the ratio between the quantity of stabilizer and of the nonstabilizer (i.e. the sc and the passive material). For consistency, the critical current of the superconductor is defined as the critical current over the non-Cu cross section.

In Table I we give $\nu_{\mathrm{Cu} \text {-sc }}$ for cables of 10 dipoles that have been built in the past 30 years, plus the NED design. Seven of them (Tevatron [1], HERA [2], the Superconducting Super Collider (SSC) $50 \mathrm{~mm}$ dipole [3], the Relativistic Heavy Ion Collider (RHIC) [4], the LHC dipole [5,6], and CERN FRESCA [7]) use Nb-Ti conductors. The remaining five (CERN-Elin [8], University of Twente MSUT [9], Berkeley D20 [10], Fermilab HFDA [13], and NED [14]) use $\mathrm{Nb}_{3} \mathrm{Sn}$ conductors. The value of $\nu_{\mathrm{Cu}-\mathrm{sc}}$ ranges from 1.2 to 2 for typical cases; RHIC dipoles have rather high $\nu_{\mathrm{Cu}-\mathrm{sc}}(2.25)$, whereas very low values have been used for D20 (0.43 to 1). All the $\mathrm{Nb}_{3}$ Sn magnets plus Fresca are $1 \mathrm{~m}$ models, whereas the others are either long prototypes (SSC) or magnets belonging to a production of several hundreds units that have been used in an accelerator (Tevatron, HERA, RHIC, LHC).

Wires are then assembled in cables, to obtain conductors with high operating currents, and finally insulated. These steps bring an additional dilution of the quantity of superconductor in the winding, which can be estimated in 10\%$20 \%$ for each step (see Table I). We define $\kappa_{\mathrm{w}-\mathrm{c}}$ as the compaction factor, i.e., the ratio between the area of the strands in the conductor and the area of the bare conductor. We define $\kappa_{\mathrm{c}-\mathrm{i}}$ as the ratio between the area of the bare conductor and of the insulated conductor. The current density $j$ flowing in the insulated conductor (usually called engineering current density) is therefore given by

$$
j=\kappa_{\mathrm{w}-\mathrm{c}} \kappa_{\mathrm{c}-\mathrm{i}} \frac{j_{\mathrm{sc}}}{1+\nu_{\mathrm{Cu}-\mathrm{sc}}} \equiv \kappa j_{\mathrm{sc}}
$$

TABLE I. Filling factors for cables used in some superconducting dipoles.

\begin{tabular}{lccccc}
\hline \hline Magnet & $\nu_{\mathrm{Cu}-\mathrm{Sc}}$ & $\kappa_{\mathrm{w}-\mathrm{c}}$ & $\kappa_{\mathrm{c}-\mathrm{i}}$ & $\kappa$ & Material \\
\hline Tevatron MB & 1.85 & 0.82 & 0.81 & 0.23 & $\mathrm{Nb}-\mathrm{Ti}$ \\
HERA MB & 1.88 & 0.89 & 0.85 & 0.26 & $\mathrm{Nb}-\mathrm{Ti}$ \\
SSC MB inner & 1.50 & 0.84 & 0.89 & 0.30 & $\mathrm{Nb}-\mathrm{Ti}$ \\
SSC MB outer & 1.78 & 0.88 & 0.84 & 0.27 & $\mathrm{Nb}-\mathrm{Ti}$ \\
RHIC MB & 2.25 & 0.87 & 0.84 & 0.23 & $\mathrm{Nb}-\mathrm{Ti}$ \\
LHC MB inner & 1.65 & 0.87 & 0.87 & 0.29 & $\mathrm{Nb}-\mathrm{Ti}$ \\
LHC MB outer & 1.95 & 0.86 & 0.83 & 0.24 & $\mathrm{Nb}-\mathrm{Ti}$ \\
FRESCA inner & 1.60 & 0.87 & 0.88 & 0.29 & $\mathrm{Nb}-\mathrm{Ti}$ \\
FRESCA outer & 1.87 & 0.88 & 0.85 & 0.26 & $\mathrm{Nb}-\mathrm{Ti}$ \\
CERN-Elin inner & 1.63 & 0.88 & 0.88 & 0.29 & $\mathrm{Nb}{ }_{3} \mathrm{Sn}$ \\
CERN-Elin outer & 1.78 & 0.87 & 0.84 & 0.26 & $\mathrm{Nb}_{3} \mathrm{Sn}$ \\
MSUT inner & 1.25 & 0.85 & 0.88 & 0.33 & $\mathrm{Nb}_{3} \mathrm{Sn}$ \\
MSUT outer & 1.25 & 0.91 & 0.85 & 0.34 & $\mathrm{Nb}_{3} \mathrm{Sn}$ \\
LBNL D20 inner & 0.43 & 0.83 & 0.84 & 0.48 & $\mathrm{Nb}_{3} \mathrm{Sn}$ \\
LBNL D20 outer & 1.00 & 0.88 & 0.77 & 0.34 & $\mathrm{Nb}_{3} \mathrm{Sn}$ \\
FNAL HFDA02-03 & 1.25 & 0.86 & 0.76 & 0.29 & $\mathrm{Nb}_{3} \mathrm{Sn}$ \\
NED & 1.25 & 0.83 & 0.84 & 0.31 & $\mathrm{Nb}_{3} \mathrm{Sn}$ \\
\hline \hline
\end{tabular}


where we defined the filling factor $\kappa$ which ranges from $1 / 3$ to $1 / 4$ in typical cases, reaching nearly 0.5 for the D20 inner layer conductor (see Table I).

The fit for the critical surface for the engineering current density can then be written as

$$
\begin{array}{ll}
j_{c}=\kappa c(b-B) & \text { for } \mathrm{Nb}-\mathrm{Ti}, \\
j_{c}=\kappa c\left(\frac{b}{B}-1\right) & \text { for } \mathrm{Nb}_{3} \mathrm{Sn},
\end{array}
$$

where in both cases $B<b$.

\section{B. Critical field, current, and peak field}

We now consider a dipole coil cross section, i.e., a layout of conductors that satisfies a twofold symmetry and where the current is flowing in opposite directions in each adjacent coil (see Fig. 2, where a $60^{\circ}$ sector coil is shown).

We assume that the magnetic field is entirely given by the current lines, that there is no contribution given by the iron, and that the current density $j$ in the coil is uniform. The current density is defined as the conductor current divided by the cross-sectional surface of the insulated conductor. We then define: (i) the field $B[\mathrm{~T}]$ at the center of the dipole; (ii) the peak field $B_{p}$ [T], i.e., the highest value (in module) of the magnetic field in the coil. One can prove that, for uniform $j$, the maximum of the field is on the contour line of the coils.

Because of the linearity of the Biot-Savart law, both $B$ and $B_{p}$ are proportional to the current density in the coil $j$ :

$$
\begin{gathered}
B=j \gamma \\
B_{p}=j \lambda \gamma,
\end{gathered}
$$

where we defined the following parameters that characterize the coil layout: (i) $\gamma\left[\mathrm{T} \mathrm{m}^{2} / \mathrm{A}\right]$ is the central field (in T) per unit of current density (in $\mathrm{A} / \mathrm{m}^{2}$ ); (ii) $\lambda$ [adim] is the ratio between the peak field and the central field.

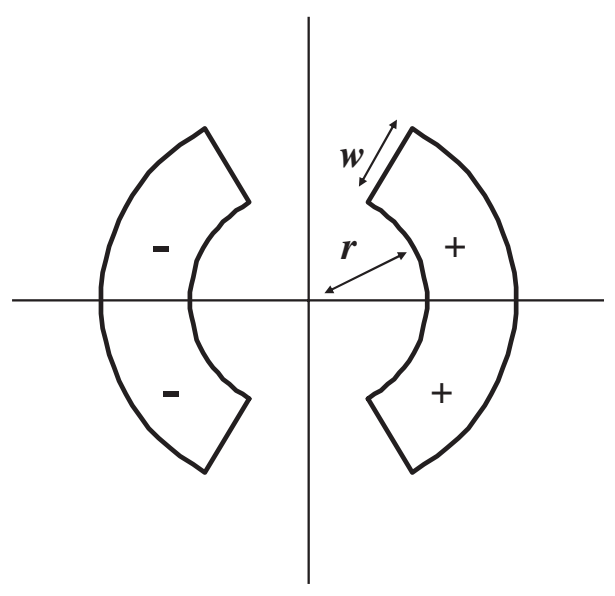

FIG. 2. Layout of a $60^{\circ}$ sector coil for a dipole of aperture radius $r$ and coil width $w$.
For the $\mathrm{Nb}-\mathrm{Ti}$, substituting Eq. (6) in Eq. (8) we can solve for the critical peak field $B_{p, c}$, which is reached in the coil when the critical surface is hit (see Fig. 3):

$$
B_{p, c}=\frac{\kappa c b}{1+\kappa c \lambda \gamma} \lambda \gamma
$$

This corresponds to a point on the critical surface $j_{p, c}$,

$$
j_{p, c}=\frac{\kappa c b}{1+\kappa c \lambda \gamma}
$$

which gives the maximum obtainable field in the center of the dipole,

$$
B_{\mathrm{ss}}=\frac{\kappa c b}{1+\kappa c \lambda \gamma} \gamma
$$

$B_{\text {ss }}$ means central field at the short sample limit (i.e. the experimental evaluation of the superconductor critical surface) and it is sometimes improperly called quench field. Indeed, the actual quench is also determined by the stability vs perturbation, i.e., by the induced disturbance, conductor design, and by the cooling conditions of the coil. Following the jargon currently in use, we denote the quantity defined in (11) by short sample field. The denomination "magnet critical field" would be more appropriate, but it would lead to ambiguity with the critical field of the superconducting material $B^{*}{ }_{c 2}$.

For the $\mathrm{Nb}_{3} \mathrm{Sn}$, using the fit (6) and Eqs. (7) and (8), one can derive

$$
\begin{aligned}
j_{p, c} & =\frac{\kappa c}{2}\left(\sqrt{\frac{4 b}{\kappa c \lambda \gamma}+1}-1\right) \\
B_{p, c} & =\frac{\kappa c \lambda \gamma}{2}\left(\sqrt{\frac{4 b}{\kappa c \lambda \gamma}+1}-1\right)
\end{aligned}
$$

$$
B_{\mathrm{ss}}=\frac{\kappa c \gamma}{2}\left(\sqrt{\frac{4 b}{\kappa c \lambda \gamma}+1}-1\right)
$$

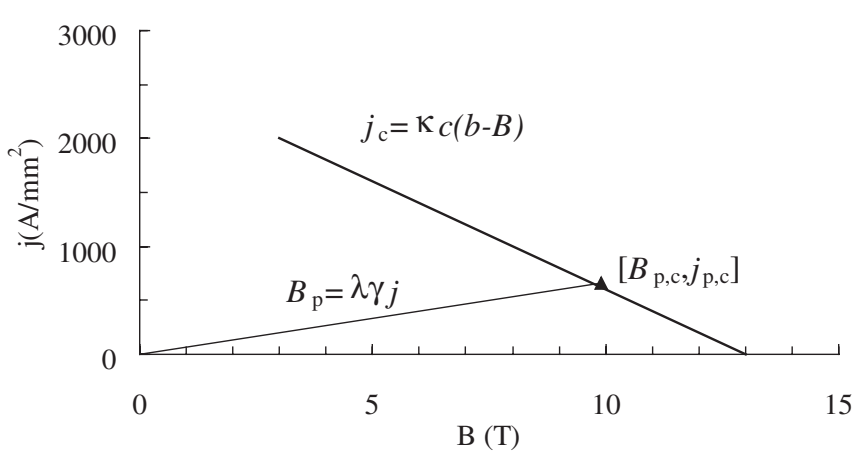

FIG. 3. Example of critical surface, loadline, critical current, and critical peak field for the LHC main dipole ( $\mathrm{Nb}-\mathrm{Ti}$ at $1.9 \mathrm{~K})$. 


\section{Field limited and current limited regimes}

The previous formulas (9)-(11) suggest that for the NbTi there are two distinct regimes.

(i) $\kappa c \lambda \gamma \ll 1$. - The critical current at short sample field (10) is equal to $\kappa c b$, i.e., it depends only on the superconducting properties and it is independent of the coil layout. Moreover, the short sample field $B_{\mathrm{ss}}$ is equal to $\kappa c \gamma b$ and is independent of $\lambda$. When we add more cable, the corresponding increase of $\gamma$ directly affects $B_{\mathrm{ss}}$. An increase of the filling ratio $\kappa$ also directly affects $B_{\mathrm{ss}}$. The magnetic field is low, and therefore superconducting coils are in general not used for these cases. Moreover, the linear approximation for the critical surface is not valid any more. Anyway, this regime (denoted as current limited) has some interest as a limiting case.

(ii) $\kappa c \lambda \gamma \gg 1$. - In this case the critical peak field tends to $b$, and the critical current tends to zero. All quantities become independent of $\kappa$. The short sample field is

$$
B_{\mathrm{ss}} \approx \frac{b}{\lambda}
$$

and the behavior of $\lambda$ for large coils determines its maximum value. We denote this regime as field limited.

For the $\mathrm{Nb}_{3} \mathrm{Sn}$, the relevant quantity is $\kappa c \lambda \gamma /(4 b)$; when $\kappa c \lambda \gamma /(4 b) \gg 1$ one has a regime which is similar to the field limited case for the $\mathrm{Nb}$ - Ti, where the critical current density tends to zero and one obtains the same result as in Eq. (15).

In Table II we give the aperture and the factors $\kappa c \lambda \gamma$ and $\kappa c \lambda \gamma /(4 b)$ for 10 dipoles that have been built in the past 30 years, plus NED. For each layout we computed the factors for the conductor $\left(\mathrm{Nb}-\mathrm{Ti}\right.$ or $\left.\mathrm{Nb}_{3} \mathrm{Sn}\right)$ used in the magnet. Since the aim is to analyze the design and not the improvements of the cable performance, here we choose the same cable properties for magnets with the same material. Some layouts are close to the field limited regime for the $\mathrm{Nb}-\mathrm{Ti}$ (in particular, Fresca has a $\kappa c \lambda \gamma$ larger than

TABLE II. Current/field limited factors for $\mathrm{Nb}-\mathrm{Ti}$ and $\mathrm{Nb}_{3} \mathrm{Sn}$ for some superconducting dipoles.

\begin{tabular}{|c|c|c|c|c|c|}
\hline Name & $\begin{array}{l}\text { Aperture } \\
(\mathrm{mm})\end{array}$ & $\begin{array}{c}\text { Coil } \\
\text { width } \\
(\mathrm{mm})\end{array}$ & $\begin{array}{c}\kappa \\
(\operatorname{adim}) \\
\end{array}$ & $\begin{array}{c}\mathrm{Nb}-\mathrm{Ti} \\
\kappa c \lambda \gamma \\
(\text { adim) }\end{array}$ & $\begin{array}{c}\mathrm{Nb}_{3} \mathrm{Sn} \\
\kappa c \lambda \gamma /(4 b) \\
(\operatorname{adim})\end{array}$ \\
\hline Tevatron MB & 38.05 & 16 & 0.232 & 1.5 & \\
\hline HERA MB & 37.50 & 21 & 0.262 & 2.1 & \\
\hline SSC MB & 25.00 & 26 & 0.298 & 3.1 & \\
\hline RHIC MB & 40.00 & 10 & 0.226 & 1.0 & \\
\hline LHC MB & 28.00 & 31 & 0.286 & 3.5 & \\
\hline FRESCA & 43.90 & 34 & 0.293 & 4.2 & \\
\hline CERN-Elin & 27.50 & 34 & 0.293 & & 0.35 \\
\hline MSUT & 24.95 & 39 & 0.330 & & 0.47 \\
\hline LBNL D20 & 25.00 & 53 & 0.484 & & 0.89 \\
\hline FNAL HFDA02-03 & 21.75 & 29 & 0.288 & & 0.22 \\
\hline NED-II & 44.00 & 53 & 0.309 & & 0.44 \\
\hline
\end{tabular}

4), i.e. $\sim 10 \mathrm{~T}$ at $1.9 \mathrm{~K}$. On the other hand, none of the designs are close to the field limited regime for the $\mathrm{Nb}_{3} \mathrm{Sn}$, i.e., thicker coils would give a sizable increase in the magnetic field. This means that $\mathrm{Nb}_{3} \mathrm{Sn}$ magnets which have been built in the past are still relatively far from the ultimate limits of this material, i.e. $\sim 20 \mathrm{~T}$.

\section{ANALYSIS OF THE CIRCULAR SHELL (SECTOR) LAYOUT}

\section{A. Coil layout description}

We first consider a shell design composed by one sector of radial width $w$, of $60^{\circ}$ azimuthal width, at a distance $r$ from the aperture center (see Fig. 2). This well-known textbook example sets to zero the field harmonic $b_{3}$ (see Appendix B).

If we use a wedge in the sector, we have three parameters: the angle $\alpha_{1}$ of the upper edge of the first block, and the angles $\alpha_{2}$ and $\alpha_{3}$ of the lower and of the upper edge of the second block (see Fig. 4, left). We assume that there is no wedge in the midplane, i.e., that the angle of the lower edge of the first block is zero. One can prove that there is a one-parameter family of solutions that set $b_{3}=b_{5}=0$ (see Appendix B). Among them we analyze $\left(\alpha_{1}, \alpha_{2}, \alpha_{3}\right)=$ $\left(48^{\circ}, 60^{\circ}, 72^{\circ}\right)$ and the unique solution $\sim\left(43.2^{\circ}, 52.2^{\circ}\right.$, $67.3^{\circ}$ ) that sets $b_{3}=b_{5}=b_{7}=0$ (see Fig. 4, left). With two wedges (three blocks) one has five parameters, and one can prove that there is one solution $\left(\alpha_{1}, \alpha_{2}, \alpha_{3}, \alpha_{4}, \alpha_{5}\right) \sim$ $\left(33.3^{\circ}, 37.1^{\circ}, 53.1^{\circ}, 63.4^{\circ}, 71.8^{\circ}\right)$ that sets to zero all odd multipoles up to $b_{11}$ (see Fig. 4, right).

Summarizing, these are the four cases of the shell design we are going to study: (i) one block: the $\left[0^{\circ}-60^{\circ}\right]$ sector (one layer, no copper wedge, $b_{3}=0$ ); (ii) two blocks: the $\left[0^{\circ}-48^{\circ}, 60^{\circ}-72^{\circ}\right]$ sectors $\left(b_{3}=b_{5}=0\right)$; (iii) two blocks: the $\left[0^{\circ}-43.2^{\circ}, 52.2^{\circ}-67.3^{\circ}\right]$ sectors $\left(b_{3}=b_{5}=\right.$ $b_{7}=0$ ); (iv) three blocks: the $\left[0^{\circ}-33.3^{\circ}, 37.1^{\circ}-53.1^{\circ}\right.$, $\left.63.4^{\circ}-71.8^{\circ}\right]$ sectors $\left(b_{3}=b_{5}=b_{7}=b_{9}=b_{11}=0\right)$.

For completeness, we also consider the case of a sector at $90^{\circ}$ with a current density proportional to the cosine of the azimuthal position ( $\cos \theta \operatorname{coil})$, providing a pure dipolar field. Its features have been analyzed in detail in $[18,19]$.

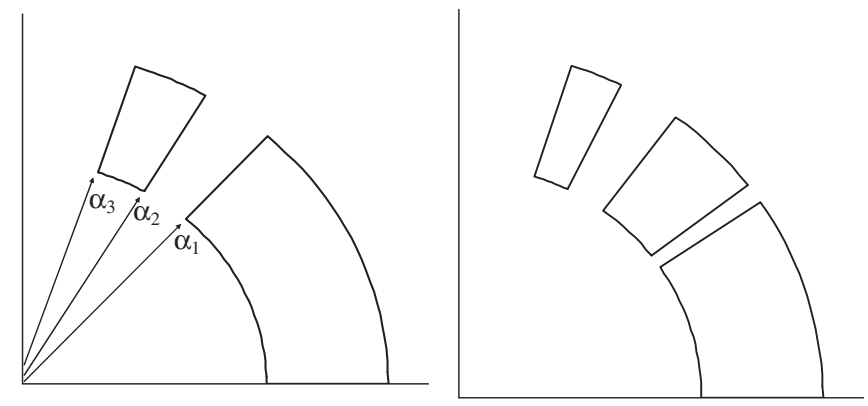

FIG. 4. Two sector layouts (one fourth shown in the plot), one with two blocks $\left[0^{\circ}-43.2^{\circ}, 52.2^{\circ}-67.3^{\circ}\right]$ (left) and one with three blocks $\left[0^{\circ}-33.3^{\circ}, 37.1^{\circ}-53.1^{\circ}, 63.4^{\circ}-71.8^{\circ}\right]$ (right). 
TABLE III. Values of parameter $\gamma_{0}$ defined in Eq. (17) for different sector coil layouts.

\begin{tabular}{lll}
\hline \hline Number of blocks & \multicolumn{1}{c}{ Block angles } & $\gamma_{0}[\mathrm{~T} \mathrm{~m} / \mathrm{A}]$ \\
\hline 1 & {$\left[0^{\circ}-60^{\circ}\right]$} & $6.93 \times 10^{-7}$ \\
2 & {$\left[0^{\circ}-48^{\circ}, 60^{\circ}-72^{\circ}\right]$} & $6.63 \times 10^{-7}$ \\
2 & {$\left[0^{\circ}-43.2^{\circ}, 53.2^{\circ}-67.3^{\circ}\right]$} & $6.53 \times 10^{-7}$ \\
3 & {$\left[0^{\circ}-33.3^{\circ}, 37.1^{\circ}-53.1^{\circ}, 63.4^{\circ}-71.8^{\circ}\right]$} & $6.48 \times 10^{-7}$ \\
& $\cos \theta$ & $6.28 \times 10^{-7}$ \\
\hline \hline
\end{tabular}

\section{B. Evaluation of the central field}

The computation of the central field for the sector layout is straightforward. For a sector of angular width $\alpha$, one has (see Appendix A)

$$
B \propto \int_{r}^{r+w} \int_{0}^{\alpha} \frac{\rho d \rho d \theta}{\rho e^{-i \theta}} \propto w \sin (\alpha)
$$

and therefore in the case of one layer made of sectors of width $w$, one has

$$
B=j \gamma_{0} w \quad \gamma=\gamma_{0} w
$$

where the constant $\gamma_{0}$ depends on the layout (see Table III).

\section{Evaluation of the peak field}

The evaluation of the ratio between peak field and central field $\lambda$ is less straightforward than the previous case. The dependence of $\lambda$ on $w$ for an aperture radius $r$ of $30 \mathrm{~mm}$ has been evaluated using a numerical computation (see Fig. 5). The shapes of the curves are very similar in the four layouts: for increasing $w, \lambda$ decreases, tending to an asymptotic value close to 1 . The difference between the layouts is $6 \%$ at $w=20 \mathrm{~mm}, 4 \%$ at $w=40 \mathrm{~mm}$, and disappears for large $w$. One can prove that the parameter $\lambda$ is a function of $w / r$. A good fit is

$$
\lambda(w, r)=1+\frac{a r}{w} .
$$

For the $\left[0^{\circ}-48^{\circ}, 60^{\circ}-72^{\circ}\right]$ case, the fit with $a=0.06$ is accurate within $2 \%$ for $w>8 \mathrm{~mm}$, and within $0.5 \%$ for $w>14 \mathrm{~mm}$. The $\cos \theta$ coil has $\lambda=1$.

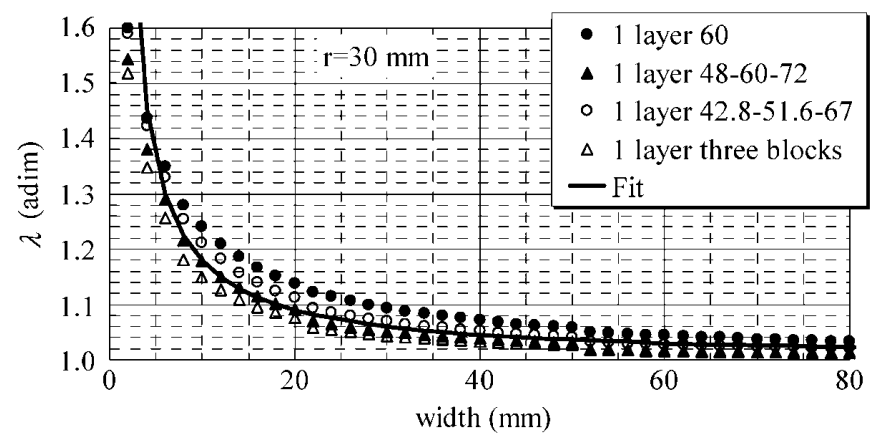

FIG. 5. Numerical evaluation of $\lambda$ and fit defined in Eq. (18) versus sector width for different sector layouts, aperture radius of $30 \mathrm{~mm}$.

\section{Short sample field versus layouts and analytical fit}

The comparison of the short sample field versus the sector width (see Fig. 6) for the $\mathrm{Nb}$-Ti shows that all the analyzed sector layouts give the same $B_{\text {ss }}$ within $1.5 \%$ for $w>6 \mathrm{~mm}$. On the other hand, the $\cos \theta$ coil gives a $B_{\mathrm{ss}}$ larger by $10 \%$ for small $w$, that reduces to $3 \%$ for $w=$ $30 \mathrm{~mm}$, and converges to the sector values for $w>30 \mathrm{~mm}$. If $B_{\mathrm{ss}}$ is expressed in terms of the coil cross-sectional area, the difference between the layouts is further reduced. This shows that for the four analyzed sector coils the presence of a copper wedge, its angular position, and the presence of one or two layers do not affect much $B_{\mathrm{ss}}$. A similar result holds for the $\mathrm{Nb}_{3} \mathrm{Sn}$ case.

We then propose a simple analytical approximation of the short sample field $B_{\text {ss }}$ as a function of the different parameters for the $\left[0^{\circ}-48^{\circ}, 60^{\circ}-72^{\circ}\right]$ case. We use Eq. (11), replacing $\gamma$ with its analytical expression (17), and we approximate $\lambda$ with (18), thus obtaining

$$
B_{\mathrm{ss}} \sim \frac{\kappa c B_{c 2}^{*} \gamma_{0} w}{1+\kappa c \gamma_{0} w\left(1+\frac{a r}{w}\right)}=\frac{\kappa c B_{c 2}^{*} \gamma_{0} w}{1+\kappa c \gamma_{0}(w+a r)}
$$

with $\gamma_{0}=0.663 \times 10^{-6}[\mathrm{Tm} / \mathrm{A}], a=0.06$ and $w, r$ expressed in meters. We then express $w$ in terms of the conductor cross-sectional area: since for the $\left[0^{\circ}-48^{\circ}\right.$, $60^{\circ}-72^{\circ}$ ] case

$$
A=\frac{2 \pi}{3}\left[(r+w)^{2}-r^{2}\right]
$$

one has

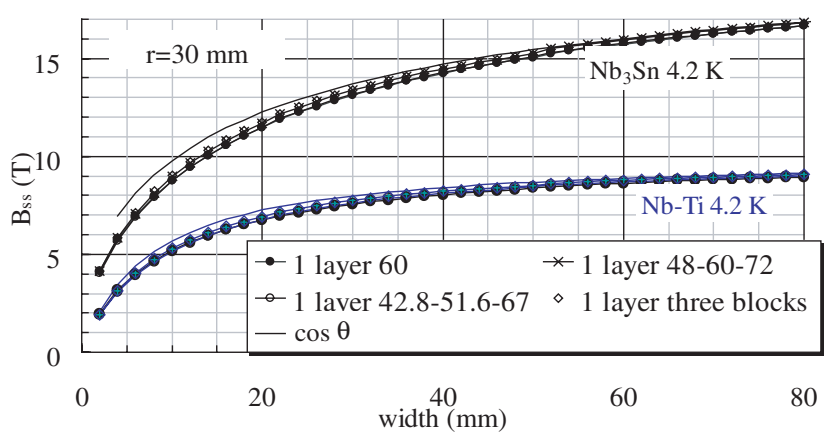

FIG. 6. (Color) Numerical evaluation of the short sample field versus sector width for different sector layouts, aperture radius of $30 \mathrm{~mm}$. 


$$
w=r\left(\sqrt{1+\frac{3 A}{2 \pi r^{2}}}-1\right)
$$

and therefore we finally obtain

$$
B_{\mathrm{ss}} \sim \frac{\kappa c B_{c 2}^{*} \gamma_{0} r\left(\sqrt{1+\frac{3 A}{2 \pi r^{2}}}-1\right)}{1+\kappa c r \gamma_{0}\left[\sqrt{1+\frac{3 A}{2 \pi r^{2}}}-1+a\right]} .
$$

The approximation agrees with the numerical values of the four considered layouts within $3 \%$ for $w>8 \mathrm{~mm}$.

Since the conductor area is a quantity which is not easy to appreciate, for a generic coil layout characterized by $A$ and $r$ we define an aspect ratio $w_{\text {eq }} / r$ where $w_{\text {eq }}$ is the width of a $60^{\circ}$ sector coil with the same area

$$
w_{\mathrm{eq}} \equiv\left(\sqrt{1+\frac{3 A}{2 \pi r^{2}}}-1\right) r
$$

and in the following sections we will express the results as a function of the aspect ratio $w_{\text {eq }} / r$ rather than in terms of coil surface $A$. For the $\mathrm{Nb}_{3} \mathrm{Sn}$ case, using Eq. (14) one obtains

$$
B_{\mathrm{ss}} \sim \frac{\kappa c \gamma_{0} w_{\mathrm{eq}}}{2}\left(\sqrt{\frac{4 b}{\kappa c \gamma_{0}\left(w_{\mathrm{eq}}+a r\right)}+1}-1\right) .
$$

\section{E. Short sample field versus sector width}

The analytical approximations we derived for the short sample field $B_{\mathrm{ss}}$ in a sector coil (22) and (24) have a strong dependence on the coil width $w$, and a weaker dependence on the aperture radius $r$. A smaller aperture gives a $\lambda$ closer to one [see Eq. (18)], and therefore a higher $B_{\mathrm{ss}}$. For small aperture radius, the sector results tend to the $\cos \theta \operatorname{coil}$. For large aperture radius, the sector results are smaller than the $\cos \theta$ coil (see Fig. 7). The ratio between the short sample field $B_{\text {ss }}$ and $B^{*}{ }_{c 2}$ that can be obtained with a sector width $w$ as a function of the aperture radius is given for the $\mathrm{Nb}-\mathrm{Ti}$ in Tables IV and V for two typical values of the filling factor: for $\kappa=0.35$, a coil width of $15 \mathrm{~mm}$ gives at most $2 / 3$ of the critical field, $80 \%$ at $w=30 \mathrm{~mm}$ and $90 \%$ at

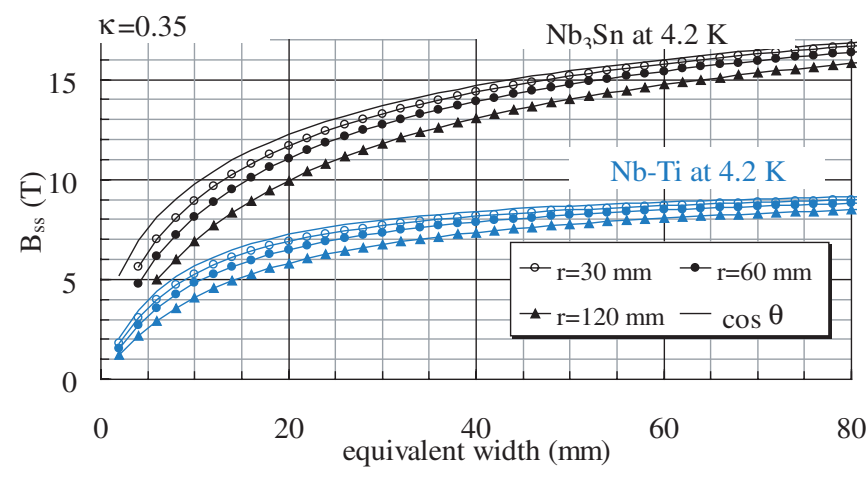

FIG. 7. (Color) Short sample field versus equivalent width according to (22) and (24) for $\kappa=0.35$.
TABLE IV. Ratio between the short sample field evaluated through Eq. (22) and $B_{c 2}^{*}$ for $\mathrm{Nb}-\mathrm{Ti}$, different sector widths and aperture radii, for filling factor $\kappa=0.35$.

\begin{tabular}{llllll}
\hline \hline & \multicolumn{5}{c}{$w(\mathrm{~mm})$} \\
& 15 & 30 & 45 & 60 & 90 \\
\hline cos theta & 0.66 & 0.80 & 0.86 & 0.89 & 0.92 \\
$r=10 \mathrm{~mm}$ & 0.66 & 0.79 & 0.85 & 0.89 & 0.92 \\
$r=30 \mathrm{~mm}$ & 0.63 & 0.77 & 0.83 & 0.87 & 0.91 \\
$r=60 \mathrm{~mm}$ & 0.58 & 0.74 & 0.81 & 0.85 & 0.89 \\
$r=120 \mathrm{~mm}$ & 0.51 & 0.68 & 0.76 & 0.81 & 0.86 \\
\hline \hline
\end{tabular}

TABLE V. Ratio between the short sample field evaluated through Eq. (22) and $B_{c 2}^{*}$ for $\mathrm{Nb}-\mathrm{Ti}$, different sector widths and aperture radii, for filling factor $\kappa=0.25$.

\begin{tabular}{llllll}
\hline \hline & \multicolumn{5}{c}{$w(\mathrm{~mm})$} \\
& 15 & 30 & 45 & 60 & 90 \\
\hline cos theta & 0.59 & 0.74 & 0.81 & 0.85 & 0.89 \\
$r=10 \mathrm{~mm}$ & 0.58 & 0.74 & 0.81 & 0.85 & 0.89 \\
$r=30 \mathrm{~mm}$ & 0.56 & 0.72 & 0.79 & 0.84 & 0.88 \\
$r=60 \mathrm{~mm}$ & 0.52 & 0.69 & 0.77 & 0.81 & 0.87 \\
$r=120 \mathrm{~mm}$ & 0.47 & 0.63 & 0.72 & 0.78 & 0.84 \\
\hline \hline
\end{tabular}

$w=60 \mathrm{~mm}$. These percentages are decreasing for increasing radii, and for smaller filling factors.

\section{F. The one-layer layout without field quality}

We then consider a sector of angular width ranging from $40^{\circ}$ to $80^{\circ}$. In this case we neglect all aspects related to field quality, which will be not optimum except in the case of $60^{\circ}$ for $b_{3}$, i.e., we assume that the field harmonics can be compensated by corrector magnets. The aim of the simulation is to verify if relaxing the field quality constraint one can improve $B_{\mathrm{ss}}$. In Fig. 8 we show for an aperture of $30 \mathrm{~mm}$ and for $\mathrm{Nb}$-Ti that a sector of $50^{\circ}$ to $60^{\circ}$ is the optimum solution, the other sectors providing a smaller critical field for the same conductor surface. A

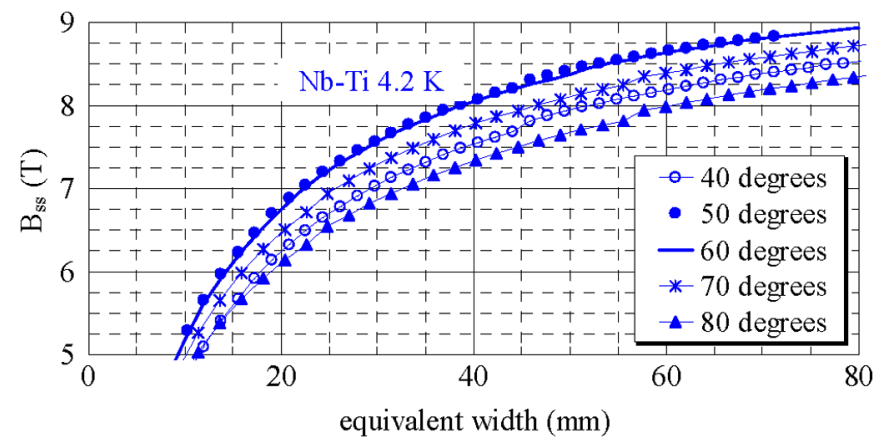

FIG. 8. (Color) Short sample field versus equivalent sector width for different angular widths of the sectors for an aperture radius of $30 \mathrm{~mm}, \mathrm{Nb}-\mathrm{Ti}$ case. Note that $50^{\circ}$ and $60^{\circ}$ curves are overlapping. 
similar result holds for the $\mathrm{Nb}_{3} \mathrm{Sn}$. Unfortunately, no theoretical justification of this numerical result is available.

\section{G. Graded coils}

In the analyzed cases of sector coils, the peak field is located in the inner part of the coil, close to the pole. The outer part of the coil has a much lower field and therefore a higher current density could be used. The technique of using a higher current density in the coil regions that are far from the location of the peak field is called grading. It allows one to reach either a highest field for the same coil area, or the same field but with a smaller coil area. The equations for computing the short sample field in the case of grading are given in Appendix C.

We carried out a simulation for a $30 \mathrm{~mm}$ aperture radius sector coil with a two blocks inner layer canceling $b_{3} b_{5}$, and $b_{7}$ (i.e., the $\left[0^{\circ}-43.2^{\circ}, 52.2^{\circ}-67.3^{\circ}\right]$ solution), and a $60^{\circ}$ outer layer of the same width. We varied the layer width from 5 to $40 \mathrm{~mm}$, we selected a filling factor of 0.35 for the inner and 0.25 for the outer layer, and for each case we varied the ratio between the current density in the outer and in the inner layer $\left[\chi_{2}\right.$ in Eqs. $(\mathrm{C} 1)$ and $\left.(\mathrm{C} 2)\right]$ to obtain the largest short sample field. Results for the $\mathrm{Nb}-\mathrm{Ti}$ at $4.2 \mathrm{~K}$ are given in Fig. 9, where $B_{\mathrm{ss}}$ is given as a function of the equivalent coil width defined in (23). If the comparison with a nongraded case is carried out for the same coil area, the gain in $B_{\mathrm{ss}}$ given by grading is between $4 \%$ and $6 \%$, with a mild dependence on the coil width. On the other hand, if we aim at a given $B_{\mathrm{ss}}$, the save in the equivalent width of a graded coil with respect to a nongraded one is relevant: for $\mathrm{Nb}-\mathrm{Ti}$ at $4.2 \mathrm{~K}$ we have $20 \%$ at $7 \mathrm{~T}, 25 \%$ at $8 \mathrm{~T}$, and $30 \%$ at $8.5 \mathrm{~T}$. For instance, $8 \mathrm{~T}$ can be reached with an equivalent coil width of $40 \mathrm{~mm}$ without grading and of $30 \mathrm{~mm}$ with grading. We also varied the ratio between the width of the inner and the outer layer: beside the case of equal widths $w_{2}=w_{1}$ we considered thinner outer layers with $w_{2}=w_{1} / 2$ and $w_{2}=3 / 4 w_{1}$, finding similar results (see Fig. 9).

We then selected the case with $w_{2}=w_{1}$ and we varied the aperture radius from $30 \mathrm{~mm}$ to $60 \mathrm{~mm}$ and $120 \mathrm{~mm}$.

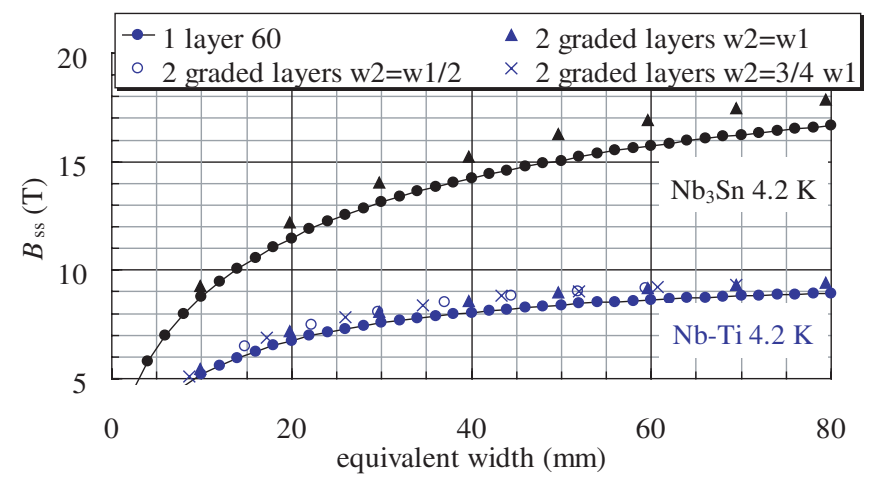

FIG. 9. (Color) Short sample field versus equivalent sector width for an aperture radius of $30 \mathrm{~mm}, \mathrm{Nb}-\mathrm{Ti}$ and $\mathrm{Nb}_{3} \mathrm{Sn}$ case, graded versus nongraded coils.
The gain in the short sample field is rather similar, i.e., $4 \%-5 \%$, becoming smaller for larger apertures. The optimal grading, providing the highest short sample field, strongly depends on the coil width and apertures. Results for the $\mathrm{Nb}-\mathrm{Ti}$ and equal widths $w_{2}=w_{1}$ are given in Fig. 10. The optimal grading is a linear function of the coil width, and the slope decreases for larger apertures (se Figs. 10 and 11). For instance, a $30 \mathrm{~mm}$ aperture radius dipole with two layers of $15 \mathrm{~mm}$ (i.e., an equivalent width of $\sim 30 \mathrm{~mm}$, similar to the main LHC dipoles) has an optimal grading of 1.6, i.e., the current density in the outer layer should be $60 \%$ larger than in the inner one. This must be considered as an upper limit, since the aspects related to the quench protection usually further reduce the applicable grading. For instance, the LHC main dipole has a grading of $23 \%$, leading to a higher field of $3.8 \%$.

The different form of the equations between $\mathrm{Nb}-\mathrm{Ti}$ and $\mathrm{Nb}_{3} \mathrm{Sn}$ [see (C8) and (C10)] implies that both the gain in $B_{\mathrm{ss}}$ and the optimal grading are different from the values found for the $\mathrm{Nb}$-Ti. However, the gain in $B_{\mathrm{ss}}$ for a given coil width is similar to the $\mathrm{Nb}-\mathrm{Ti}$ case, being $4 \%-7 \%$ and getting slightly smaller for larger apertures. The saving in the coil width is $22 \%-23 \%$ at $14-15 \mathrm{~T}$, and $27 \%$ at $16 \mathrm{~T}$ (see Fig. 9). The optimal grading (see Fig. 11) is smaller than for $\mathrm{Nb}$-Ti; for instance, a $30 \mathrm{~mm}$ aperture with two

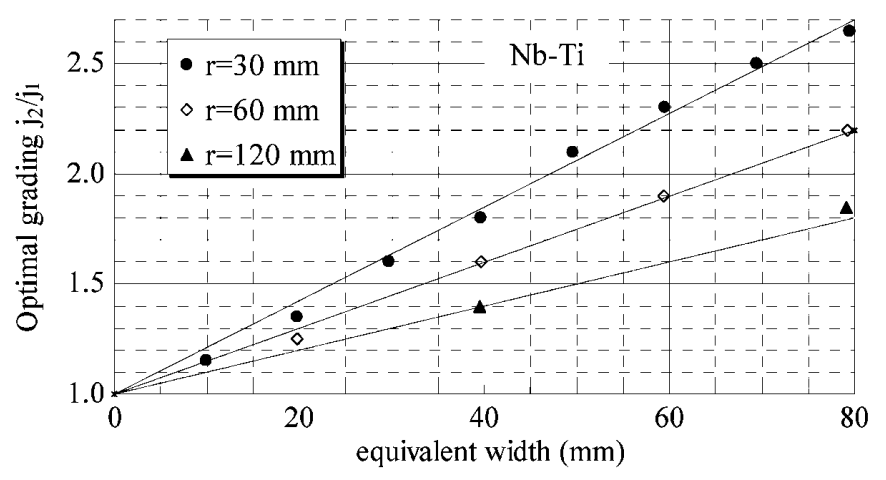

FIG. 10. Optimal grading, providing the highest short sample field, as a function of the sector width, $\kappa=0.35$, Nb-Ti.

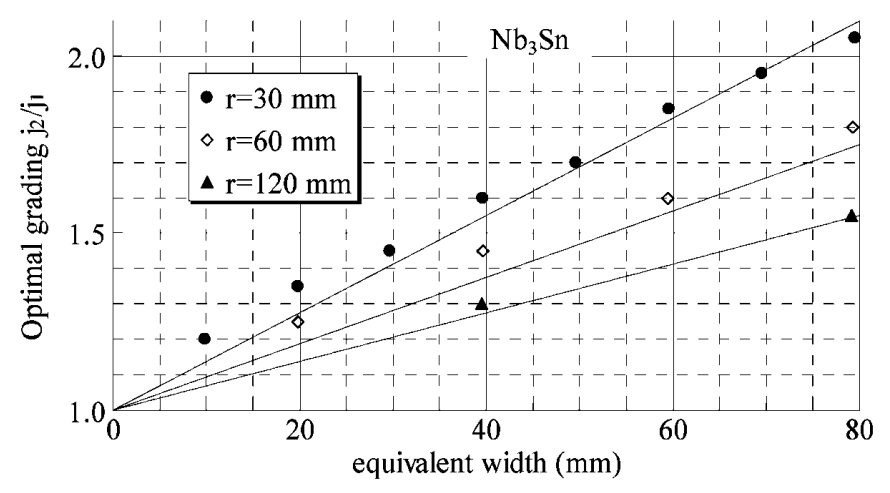

FIG. 11. Optimal grading as a function of the sector width, and $\kappa=0.35, \mathrm{Nb}_{3} \mathrm{Sn}$. 
TABLE VI. Parameters of coil layouts of 11 superconducting dipoles.

\begin{tabular}{lccccrr}
\hline \hline & $\begin{array}{c}\text { Aperture } \\
\text { radius } \\
\text { Name }\end{array}$ & & & Surface \\
$(\mathrm{mm})$ & Layers & Blocks & $\begin{array}{r}w \text { equivalent } \\
(\mathrm{mm})\end{array}$ & $\begin{array}{r}\text { Grading } \\
(\%)\end{array}$ \\
\hline Tevatron MB & 38.05 & 2 & $2[1,1]$ & 2700 & 14.3 & 0.0 \\
HERA MB & 37.50 & 2 & $4[2,2]$ & 3680 & 18.7 & 0.0 \\
SSC MB & 25.00 & 2 & $6[4,2]$ & 3224 & 21.5 & 29.9 \\
RHIC MB & 40.00 & 1 & 4 & 1723 & 9.2 & 0.0 \\
LHC MB & 28.00 & 2 & $6[4,2]$ & 4657 & 26.8 & 23.0 \\
FRESCA & 43.90 & 2 & $7[4,3]$ & 7470 & 30.2 & 23.5 \\
CERN-Elin & 27.50 & 2 & $6[4,2]$ & 5551 & 30.9 & 42.4 \\
MSUT & 24.95 & 2 & $5[3,2]$ & 6103 & 34.5 & 65.1 \\
D20 & 25.00 & 4 & $13[3,4,3,3]$ & 8998 & 45.2 & 80.5 \\
FNAL HFDA02-03 & 21.75 & 2 & $6[3,3]$ & 3253 & 23.3 & 0.0 \\
NED-II & 44.00 & 2 & $7[4,3]$ & 12712 & 45.5 & 0.0 \\
\hline \hline
\end{tabular}

layers of $15 \mathrm{~mm}$ width has an optimal grading of $45 \%$ (60\% for the $\mathrm{Nb}-\mathrm{Ti})$.

\section{H. Analysis of accelerator magnets (without iron)}

We finally compare the results of our analysis of simplified, uniform $j$ sector layouts with actual designs that have been used in accelerator magnets. In Table VI we give the main parameters of the geometry of 10 dipoles actually built in the past 30 years, plus the NED design. For each one, we compute the equivalent width $w_{\text {eq }}$ defined in Eq. (23). Apertures are ranging from 20 to $45 \mathrm{~mm}$, equivalent widths from 10 to $45 \mathrm{~mm}$, and the conductor area spans over 1 order of magnitude. All designs are based on sector coils, with one, two, or four layers, and 2 to 13 blocks. Several dipoles have grading, varying from $20 \%$ to $80 \%$. Two typical layouts are shown in Fig. 12.

The parameters $\gamma$ [see Eq. (7)] of the dipoles of Table VI, evaluated without iron, are plotted in Fig. 13 versus the equivalent width $w_{e}$ defined in Eq. (23): in the case of no current grading they all fit within $4 \%$ with the value computed for a $\left[0^{\circ}-48^{\circ}, 60^{\circ}-72^{\circ}\right]$ sector coil (note that the agreement with the $\left[0^{\circ}, 60^{\circ}\right]$ sector coil is worse, the error being $8 \%$ ). This shows that, for the analyzed cases, for a given quantity of cable one obtains within $4 \%$ the same field per unit of current density, independently of the layer or sector subdivisions. For the cases
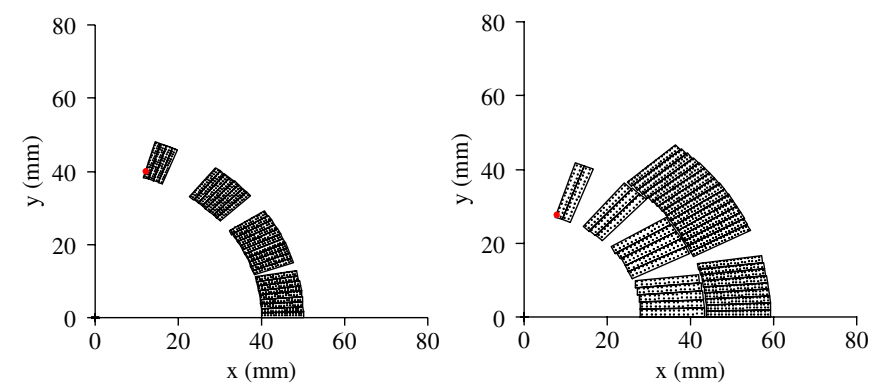

FIG. 12. Coil layout of RHIC (left) and LHC (right) dipoles. with current grading, we used the current density of the sector where the peak field is located (the inner layer in all cases) to define $\gamma$. The gain in $\gamma$ is in most analyzed cases around $20 \%$.

Results for the parameter $\lambda$ are shown in Fig. 14, where we compare the actual values of the magnets given in Table VI without iron (markers) to the results for the $\left[0^{\circ}-48^{\circ}, 60^{\circ}-72^{\circ}\right]$ sector coil (solid line). The agreement is within $1.5 \%$. Magnet data confirm the trend that $\lambda$ increases for smaller aspect ratios $w_{\text {eq }} / r$ and tends to one for the larger ones. Magnets designed with a current grading have a similar $\lambda$ to the single sector estimate.

In Table VII we give a comparison between the estimated values for the short sample field with no grading as deduced theoretically using the analytical approximations (22) and (24) with $\gamma_{0}=0.663 \times 10^{-6}[\mathrm{~T} \mathrm{~m} / \mathrm{A}]$ and $a_{0}=$ 0.06 , and the actual ones for the 11 analyzed magnets without iron. The agreement in the case of no current grading is within $1.5 \%$. One can conclude that in case of no grading Eqs. (22) and (24) model the short sample field with a high precision, neglecting the design details as the number of layers and the position of wedges. On the other

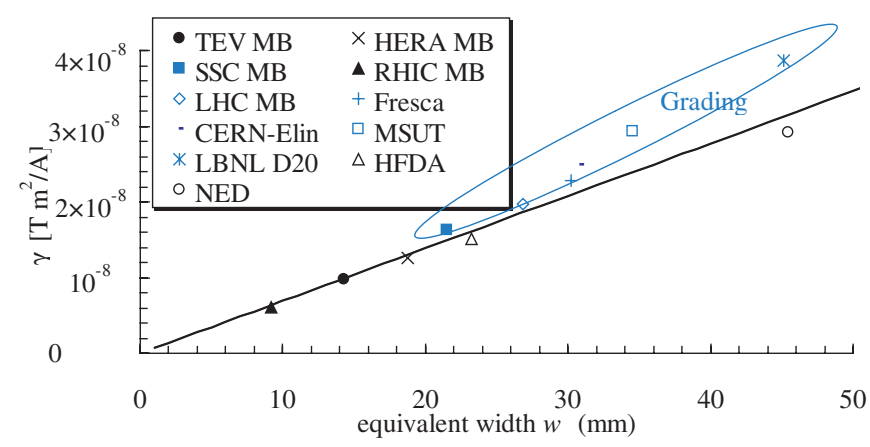

FIG. 13. (Color) Parameter $\gamma$ (central field per unit of current density) for 11 accelerator dipoles without iron (markers) and results for a $0^{\circ}-48^{\circ}, 60^{\circ}-72^{\circ}$ sector (solid line) vs equivalent width defined in Eq. (23). 


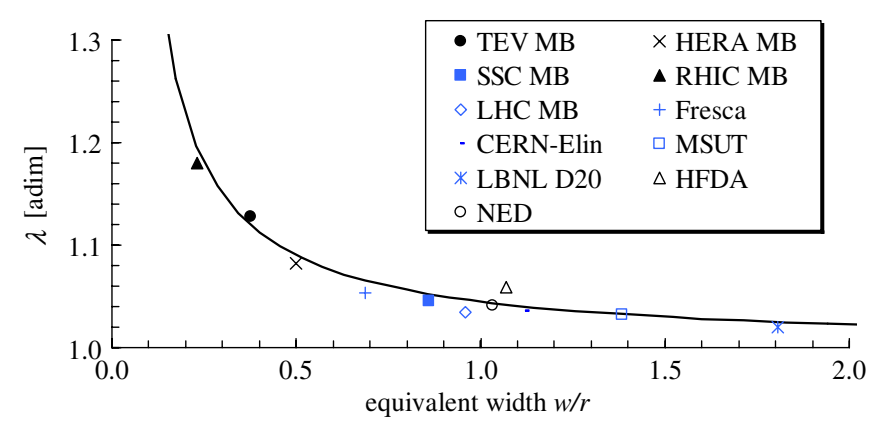

FIG. 14. (Color) Ratio $\lambda$ between peak field and current density vs equivalent ratio width/aperture radius as defined in Eq. (23): analytical fit of Eq. (18) (solid line) and values for 11 dipoles evaluated without iron (markers).

hand, 6 magnets with current grading show a higher critical field with respect to our analytical benchmark of up to $4 \%$ for $\mathrm{Nb}-\mathrm{Ti}$ and up to $7 \%$ for $\mathrm{Nb}_{3} \mathrm{Sn}$.

The above analysis confirms that for small $w / r$ the ratio peak field/central field $\lambda$ becomes less and less favorable: for instance RHIC and Tevatron magnets have a peak field $15 \%-18 \%$ larger than the central field, whereas MSUT and D20 have a peak field which is only 3\%-4\% larger than the bore field (see Fig. 14). A layout for the Gesellschaft für Schwerionenforschung (GSI) SIS-300 dipole recently proposed by the Istituto Nazionale Fisica Nucleare [23] features a rather small $w / r \sim 0.3$ but a $\lambda \sim 1.08$ well below the scaling (18). The cross section is shown in Fig. 15, left. The layout has been optimized to maximize the short sample field, given the cable width. The presence of a large wedge before the upper block, and the small number of cables of the last block on the pole, allows one to obtain a significantly lower $\lambda$, i.e., about 1.08 instead 1.16 as one would expect from the fit (18), see Fig. 15, right. This allows one to obtain (without grading) a short sample field which is $4 \%$ larger than that expected from our scaling law. One can conclude that, even though all the analyzed de-

TABLE VII. Actual and analytical estimates of critical field for 11 superconducting dipoles (no iron).

\begin{tabular}{lcccc}
\hline \hline & \multicolumn{4}{c}{ Critical field } \\
Name & $\begin{array}{c}\text { Actual } \\
(\mathrm{T})\end{array}$ & $\begin{array}{c}\text { Analytical } \\
(\mathrm{T})\end{array}$ & $\begin{array}{c}\text { Error } \\
(\%)\end{array}$ & $\begin{array}{c}\text { Grading } \\
(\%)\end{array}$ \\
\hline Tevatron MB & 5.4 & 5.3 & 1.2 & 0.0 \\
HERA MB & 6.3 & 6.2 & 1.0 & 0.0 \\
SSC MB & 7.2 & 6.9 & 4.0 & 29.9 \\
RHIC MB & 4.2 & 4.2 & 0.5 & 0.0 \\
LHC MB & 9.8 & 9.5 & 3.9 & 23.0 \\
FRESCA & 10.0 & 9.6 & 3.4 & 23.5 \\
CERN-Elin & 10.3 & 9.5 & 7.1 & 42.4 \\
MSUT & 11.2 & 10.4 & 7.4 & 65.1 \\
D20 & 13.3 & 12.5 & 6.2 & 80.5 \\
FNAL HFDA02-03 & 10.2 & 10.4 & -1.6 & 0.0 \\
NED-II & 13.8 & 13.9 & -0.6 & 0.0 \\
\hline \hline
\end{tabular}
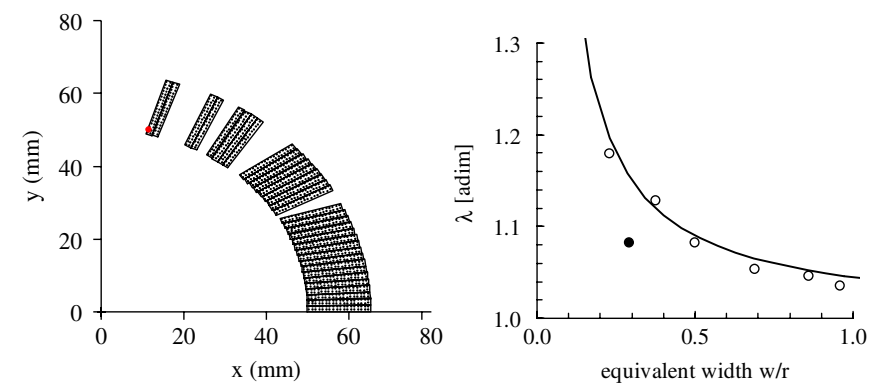

FIG. 15. Cross section of the INFN SIS-300 proposed model (left), and value of $\lambda$ (full marker) compared to the fit (18) and to numerical values (empty markers) of magnets shown in Fig. 14.

signs of built magnets agree well with the estimates based on the sector, it appears that there is still some space for further electromagnetic optimization of coil layouts with respect to what has been done in the past.

\section{IRON EFFECT}

The presence of the iron yoke has the main function of providing a return flux path shielding the external side of the magnet from the inner magnetic field. The iron also induces a higher field in the aperture for the same current density, thus improving aspects related to stability and protection. However, it also induces a higher peak field and therefore the beneficial effect on the short sample field is not as large as it can be naively expected and strongly depends on the coil width. Finally, the iron yoke can also be used to transmit the retaining forces (mechanical function). Here we will focus on its impact on the short sample field for the $\mathrm{Nb}-\mathrm{Ti}$ and $\mathrm{Nb}_{3} \mathrm{Sn}$. One has to clearly distinguish the increase of the central field for a given current density (i.e. the increase in $\gamma$ ) from the increase of the maximum obtainable field, i.e., the short sample field. The first one can be large (20\%-50\%, see Table VIII), and helps coil protection by reducing the operational current. The second one is in general rather small: rarely the iron yoke makes an increase of $10 \%$ in the short sample field and it reduces to $\sim 5 \%$ for coils larger than $30 \mathrm{~mm}$.

In order to prove these statements, we first compute the relative increase of $\gamma$ [see Eq. (7)], which is independent of the material, for the coil layouts analyzed in the previous section, see Table VIII. We then compute the gain in the short sample field. The increase is large (about $25 \%$ ) only for the RHIC dipole, where both the coil and the collar are thin. It decreases for larger coil widths, being 3\%-7\% for widths larger than $30 \mathrm{~mm}$, i.e., comparable to the effect of a strong grading. For the same layout, the increase is more relevant for $\mathrm{Nb}_{3} \mathrm{Sn}$ due to the shape of the critical surface.

\section{CONCLUSIONS}

In this paper we aimed at finding explicit expressions for the short sample field in a superconducting dipole as a function of the material and cable parameters, of the op- 
TABLE VIII. Increase of parameters $\gamma$ and $\beta$, and of the short sample field, due to the iron yoke for $7 \mathrm{Nb}$-Ti and $4 \mathrm{Nb}_{3} \mathrm{Sn}$ dipoles.

\begin{tabular}{|c|c|c|c|c|c|c|}
\hline Name & $\begin{array}{l}\text { Riron } \\
(\mathrm{mm})\end{array}$ & $\begin{array}{l}\text { Collar thickness } \\
(\mathrm{mm})\end{array}$ & $\begin{array}{l}\Delta \gamma / \gamma \\
(\%)\end{array}$ & $\Delta(\lambda \gamma) /(\lambda \gamma)$ & \multicolumn{2}{|c|}{$\begin{array}{c}\Delta B_{c} / B_{c} \\
(\%)\end{array}$} \\
\hline Tevatron $\mathrm{MB}$ & 90.0 & 36.1 & 25.2 & 22.0 & 10.4 & \\
\hline HERA MB & 86.5 & 28.2 & 29.6 & 27.1 & 9.4 & \\
\hline SSC MB & 69.0 & 19.4 & 30.9 & 29.3 & 7.2 & \\
\hline RHIC MB & 59.7 & 9.6 & 56.7 & 47.9 & 26.7 & \\
\hline LHC MB & 98.0 & 39.2 & 21.2 & 20.4 & 3.8 & \\
\hline FRESCA & 114.4 & 36.4 & 29.5 & 27.8 & 5.7 & \\
\hline CERN-Elin & 115.0 & 53.3 & 16.6 & 16.1 & & 4.1 \\
\hline MSUT & 107.0 & 43.3 & 19.8 & 19.2 & & 4.3 \\
\hline D20 & 92.5 & 14.4 & 39.9 & 39.0 & & 5.5 \\
\hline FNAL HFDA02-03 & 60.0 & 8.8 & 37.4 & 35.0 & & 10.6 \\
\hline NED-II & 130.0 & 33.2 & 31.7 & 30.4 & & 6.8 \\
\hline
\end{tabular}

erating temperature, of the magnet aperture, and of the coil width. We derived semianalytical expressions for the cases of $\mathrm{Nb}$ - $\mathrm{Ti}$ [Eq. (22)] and $\mathrm{Nb}_{3} \mathrm{Sn}$ [Eq. (24)] based on the analysis of a sector coil and making use of simple fits for the critical surfaces [Eqs. (2) and (4)]. The comparison with the numerical results relative to several nongraded dipoles built in the past 30 years show that using this method the short sample field can be estimated within a few percent.

The equation can be used as a benchmark to judge the efficiency of the magnet design: we applied this method to work out the impact of grading and the effect of the iron on the short sample field. The equation can also provide plots as shown in Fig. 7, where, having the magnetic field and aperture requirements, one can quickly estimate the needed coil thickness, without the need of going through a detailed design.

\section{ACKNOWLEDGMENTS}

We wish to acknowledge all the colleagues that helped us in recovering the data of the magnet cross sections, and, in particular, A. Den Ouden, Vadim Kashikhin, P. Ferracin, A. McInturff, S. Russenschuck, N. Schwerg, M. Sorbi, H. Ten Kate, A. Verweij, and C. Vollinger. We wish to thank J.-P. Koutchouk for useful comments on the manuscript. We acknowledge the support of the European Community-Research Infrastructure Activity under the FP6 "Structuring the European Research Area" program (CARE, Contract No. RII3-CT-2003-506395).

\section{APPENDIX A: EQUATION FOR FIELD GRADIENT AND HARMONICS}

According to the complex formalism, a line carrying a current $I$ in the position $z_{0} \equiv x_{0}+i y_{0}$ gives a magnetic field $B(z) \equiv B_{y}(z)+i B_{x}(z)$ in the position $z \equiv x+i y$ that reads

$$
B(z)=\frac{I \mu_{0}}{2 \pi\left(z-z_{0}\right)} .
$$

One can expand the series as

$$
\begin{aligned}
B(z) & =-\frac{I \mu_{0}}{2 \pi z_{0}} \sum_{n=1}^{\infty}\left(\frac{z}{z_{0}}\right)^{n-1} \\
& =-\frac{I \mu_{0}}{2 \pi z_{0}} \sum_{n=1}^{\infty}\left(\frac{R}{z_{0}}\right)^{n-1}\left(\frac{z}{R}\right)^{n-1},
\end{aligned}
$$

where $R$ is the reference radius, usually chosen as $2 / 3$ of the aperture radius. The multipolar expansion of the magnetic field according to the European notation $(n=1$ being the dipole) reads

$$
B(z)=\sum_{n=1}^{\infty} C_{n}\left(\frac{z}{R}\right)^{n-1}=\sum_{n=1}^{\infty}\left(B_{n}+i A_{n}\right)\left(\frac{z}{R}\right)^{n-1}
$$

For a perfect dipole (with a twofold symmetry) the first nonzero terms of the expansion are $B_{1}, B_{3}$, and $B_{5}$, and one can write the expansion as

$$
B(z)=B_{1}+B_{3}\left(\frac{z}{R}\right)^{2}+B_{5}\left(\frac{z}{R}\right)^{4}+\cdots
$$

or in terms of the multipoles $b_{j} \equiv 10^{4} B_{j} / B_{1}$,

$$
B(z)=B_{1}\left[1+10^{-4}\left(b_{3} \frac{z^{2}}{R^{2}}+b_{5} \frac{z^{4}}{R^{4}}+\cdots\right)\right] .
$$

In accelerator superconducting magnets the multipoles must be of the order of 1 , and must be controlled within a fraction of a unit. The first terms of (A2) are

$$
B(z)=-\frac{I \mu_{0}}{2 \pi z_{0}}-\frac{I \mu_{0}}{2 \pi\left(z_{0}\right)^{3}} z^{2}-\frac{I \mu_{0}}{2 \pi\left(z_{0}\right)^{5}} z^{4}+\cdots
$$

and therefore for a current line at $z_{0}$ one has 


$$
B_{1}=-\frac{I \mu_{0}}{2 \pi} \operatorname{Re}\left(\frac{1}{z_{0}}\right)
$$

and the non-normalized multipoles read

$$
B_{n}+i A_{n}=-\frac{I \mu_{0}}{2 \pi z_{0}} \frac{R^{n-1}}{\left(z_{0}\right)^{n-1}} .
$$

\section{APPENDIX B: FIELD QUALITY CONSTRAINTS}

We consider a dipole whose half coil layout is a sector of width $w$, from the angle $-\alpha$ to $\alpha$, at a distance $r$ from the center (see Fig. 2). The multipole coefficients can be obtained by integrating the Biot-Savart contribution of one current line [see Eq. (A7)] over the sector:

$$
B_{n}=\frac{2 \mu_{0} j R^{n-1}}{n \pi(2-n)}\left[\frac{1}{(r+w)^{n-2}}-\frac{1}{r^{n-2}}\right] \sin (n \alpha) .
$$

For $\alpha=60^{\circ}$, the first order nonzero coefficient $B_{3}$ vanishes. Since the second order nonzero coefficient $B_{5}$ is proportional to $\sin (5 \alpha)$, it becomes zero for $\alpha=36^{\circ}$ and for $\alpha=72^{\circ}$. Therefore, a single radial sector of uniform $j$ cannot have $B_{3}=B_{5}=0$.

If we consider a shell composed by two radial sectors $\left[0, \alpha_{1}\right]$ and $\left[\alpha_{2}, \alpha_{3}\right]$, i.e., with a wedge between $\alpha_{1}$ and $\alpha_{2}$, the equations for setting $B_{3}=B_{5}=0$ are

$$
\begin{aligned}
& \sin \left(3 \alpha_{3}\right)-\sin \left(3 \alpha_{2}\right)+\sin \left(3 \alpha_{1}\right)=0 \\
& \sin \left(5 \alpha_{3}\right)-\sin \left(5 \alpha_{2}\right)+\sin \left(5 \alpha_{1}\right)=0
\end{aligned}
$$

The numerical solution of Eqs. (B2) and (B3) gives a oneparameter family of solutions, shown in Fig. 16, where we used the total width of the coil as the independent parameter, ranging from $60^{\circ}$ to $90^{\circ}$. One observes that the width of the copper wedge varies from $8^{\circ}$ to $20^{\circ}$. Two typical solutions are a $72^{\circ}$ coil with a $12^{\circ}$ wedge from $48^{\circ}$ to $60^{\circ}$, and a $64^{\circ}$ coil with a $8^{\circ}$ wedge from $36^{\circ}$ to $44^{\circ}$. In Fig. 16 we also reported the value of $B_{7}$ in each case. One

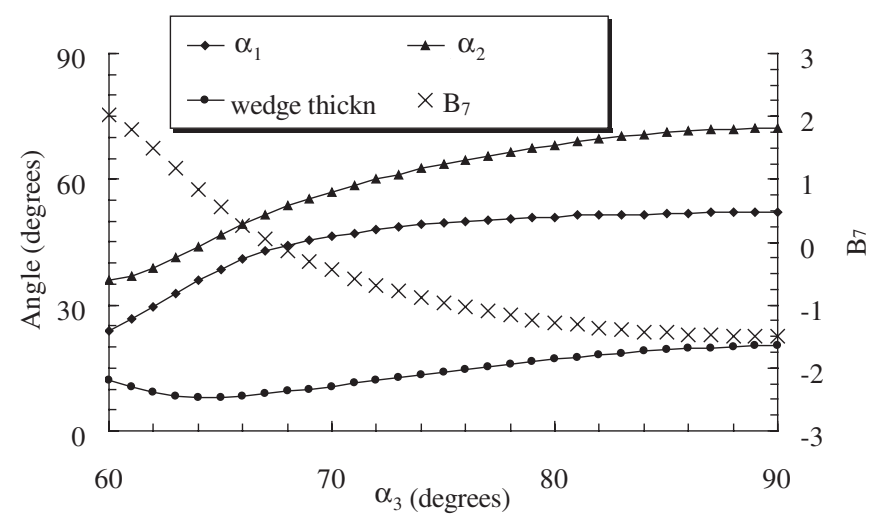

FIG. 16. One-parameter family for a single layer shell that sets $B_{3}$ and $B_{5}=0$. The angles $\alpha_{1}$ and $\alpha_{2}$ and thickness of the wedge $\alpha_{2}-\alpha_{1}$ are plotted versus $\alpha_{3}$. solution sets to zero also $B_{7}$ : the approximated values of the sector angles are $\left(43.2^{\circ}, 52.2^{\circ}, 67.3^{\circ}\right)$.

\section{APPENDIX C: EQUATIONS FOR GRADING}

We first analyze the $\mathrm{Nb}$ - Ti case. We consider a two-layer layout, where each layer $n=1,2$ has a given the current density $j_{n}$ and dilution factor $\kappa_{n}$. The field in the center is proportional to the current densities,

$$
B=j_{1} \gamma_{1}+j_{2} \gamma_{2}=j_{1}\left(\gamma_{1}+\chi_{2} \gamma_{2}\right) \equiv j_{1} \gamma,
$$

where we defined

$$
\chi_{1} \equiv \frac{j_{1}}{j_{1}}=1 \quad \chi_{2} \equiv \frac{j_{2}}{j_{1}}
$$

For each layer we can define $\gamma_{n} \lambda_{n}$ as the ratio between the peak field in that block produced by the current densities $\left(j_{1,} j_{2}\right)$ and the current density in the first block $j_{1}$,

$$
B_{p, n} \equiv j_{1} \gamma_{n} \lambda_{n}=\frac{1}{\chi_{n}} j_{n} \gamma_{n} \lambda_{n}
$$

Keeping fixed the ratio between the current densities $\chi_{2}$, we want to find the critical current density corresponding to the critical surface. In each layer, the current density must satisfy

$$
j_{c, n} \leq \kappa_{n} c\left(b-B_{p, n}\right)
$$

and substituting (C3) in (C4) and solving for $j_{c, n}$ we get

$$
j_{c, n} \leq \frac{\chi_{n} c b \kappa_{n}}{\chi_{n}+c \kappa_{n} \gamma_{n} \lambda_{n}}
$$

the above expression can be written for the current density in the first layer,

$$
j_{c, 1}=\frac{j_{c, n}}{\chi_{n}} \leq \frac{c b \kappa_{n}}{\chi_{n}+c \kappa_{n} \gamma_{n} \lambda_{n}} ;
$$

and we obtain the expression for the critical current density in the first layer,

$$
j_{c, 1}=\min _{n} \frac{c b \kappa_{n}}{\chi_{n}+c \kappa_{n} \gamma_{n} \lambda_{n}},
$$

and for the short sample field,

$$
B_{\mathrm{ss}}=\min _{n} \frac{c b \kappa_{n}}{\chi_{n}+c \kappa_{n} \gamma_{n} \lambda_{n}} \gamma
$$

Equations (C1) to (C8) can be extended to the $\mathrm{Nb}_{3} \mathrm{Sn}$ using the simplified fit we proposed in (4). One obtains

$$
j_{c, 1}=\min _{n} \frac{\kappa_{n} c}{2 \chi_{n}}\left(\sqrt{\frac{4 b \chi_{n}}{\kappa_{n} c \gamma_{n} \lambda_{n}}+1}-1\right)
$$


and for the short sample field

$$
B_{\mathrm{ss}}=\min _{n} \frac{\kappa_{n} c \gamma}{2 \chi_{n}}\left(\sqrt{\frac{4 b \chi_{n}}{\kappa_{n} c \gamma_{n} \lambda_{n}}+1}-1\right) .
$$

[1] R. Hanft et al., TM-1182, 1630, 03/1983.

[2] R. Meinke, IEEE Trans. Appl. Supercond. 27, 1728 (1991).

[3] J. Strait et al., in Particle Accelerator Conference (1991), pp. 2176-2178.

[4] M. Anerella et al., Nucl. Instrum. Methods Phys. Res., Sect. A 499, 280 (2003).

[5] R. Perin, in Encyclopedia of Applied Superconductivity (IOP, London, 1998), pp. 919-950.

[6] L. Rossi, IEEE Trans. Appl. Supercond. 13, 1221 (2003); 13, 3875 (2003).

[7] D. Leroy et al., IEEE Trans. Appl. Supercond. 10, 178 (2000).

[8] A. Asner et al., in Proceedings of MT-11 (Elsevier Applied Science, New York, 1989), pp. 36-41; also LHC Note 105 (1989).

[9] H. J. Ten Kate et al., IEEE Trans. Magn. 27, 1996 (1991).
[10] R. Benjegerdes et al., Particle Accelerator Conference (1999), pp. 3233-3235.

[11] A. R. Hafalia et al., IEEE Trans. Appl. Supercond. 14, 283 (2004).

[12] P. Ferracin et al., IEEE Trans. Appl. Supercond. 16, 378 (2006).

[13] G. Ambrosio et al., IEEE Trans. Appl. Supercond. 10, 322 (2000).

[14] A. Devred et al., Supercond. Sci. Technol. 19, S67 (2006).

[15] M.N. Wilson, Superconducting Magnets (Clarendon Press, Oxford, 1983).

[16] K. H. Mess, P. Schmuser, and S. Wolff, Superconducting Accelerator Magnets (World Scientific, Singapore, 1996).

[17] L. Rossi and E. Todesco, Phys. Rev. ST Accel. Beams 9, 102401 (2006).

[18] S. Caspi and P. Ferracin, Particle Accelerator Conference (2005), pp. 107-111.

[19] S. Caspi, P. Ferracin, and S. Gourlay, IEEE Trans. Appl. Supercond. (to be published).

[20] L. Bottura, IEEE Trans. Appl. Supercond. 10, 1054 (2000).

[21] E. J. Kramer, J. Appl. Phys. 44, 1360 (1973).

[22] S. Gourlay et al., IEEE Trans. Appl. Supercond. 16, 324 (2006).

[23] P. Bellomo, P. Fabbricatore, S. Farinon, and M. Sorbi, IEEE Trans. Appl. Supercond. (to be published). 